\title{
Cas9 targeted enrichment of mobile elements using nanopore sequencing
}

Torrin L. McDonald ${ }^{* 1}$, Weichen Zhou,2, Christopher Castro², Camille Mumm', Jessica A. Switzenberg ${ }^{2}$, Ryan E. Mills ${ }^{1,2, \dagger}$, Alan P. Boyle $e^{1,2, \dagger}$

${ }^{1}$ Department of Human Genetics, University of Michigan, Ann Arbor, MI, USA

${ }^{2}$ Department of Computational Medicine and Bioinformatics, University of Michigan, Ann Arbor, MI, USA

*These authors contributed equally

†To whom correspondence should be addressed. Email: remills@umich.edu (R.E.M.) and apboyle@umich.edu (A.P.B.)

\begin{abstract}
Mobile element insertions (MEls) are highly repetitive genomic sequences that contribute to inter- and intra-individual genetic variation and can lead to genetic disorders. Targeted and whole-genome approaches using short-read sequencing have been developed to identify reference and non-reference MEls; however, the read length hampers detection of these elements in complex genomic regions. Here, we pair Cas 9 targeted nanopore sequencing with computational methodologies to capture active MEls in human genomes. We demonstrate parallel enrichment for distinct classes of MEls, averaging $44 \%$ of reads on targeted signals. We show an individual flow cell can recover a remarkable fraction of MEls (97\% L1Hs, 93\% AluYb, 51\% AluYa, 99\% SVA_F, and 65\% SVA_E). We identify twenty-one non-reference MEls in GM12878 overlooked by modern, long-read analysis pipelines, primarily in repetitive genomic regions. This work introduces the utility of nanopore sequencing for MEl enrichment and lays the foundation for rapid discovery of elusive, repetitive genetic elements.
\end{abstract}


At least $45 \%$ of the human genome is composed of transposable element (TE)-derived sequences ${ }^{1}$. TEs can be subdivided into four major categories: (i) DNA transposons; (ii) long terminal repeat (LTR) retrotransposons; (iii) long interspersed elements (LINEs); and (iv) short interspersed elements (SINEs). L1 represents a subclass of LINEs and L1-derived sequences comprise approximately $17 \%$ of the human genome ${ }^{1,2}$. Alu elements, a subclass of SINEs, are ancestrally derived from a dimerization of the 7SL RNA gene and make up $11 \%$ of the human genome, spread out over 1 million copies ${ }^{3}$. SVA elements are active elements that have recently evolved and are derived from a SINE-R sequence coupled with a VNTR (variable number of tandem repeats) region and an Alu-like sequence ${ }^{4}$. An average human genome contains approximately 80-100 active full-length human-specific $\mathrm{L} 1 \mathrm{~s}(\mathrm{~L} 1 \mathrm{Hs})^{5-7}$ and a small number of highly active, or "hot," L1Hs sequences that are responsible for the bulk of human retrotransposition activity,5,8,9. This includes the mobilization of Alus and SVAs which require trans-acting factors from L1s to transpose ${ }^{3}$. Collectively, the result of such recent mobilization events are referred to as mobile element insertions (MEls).

Regions harboring these repetitive elements have long been considered part of the 'dark matter' of the genome with no expected impact on human phenotypes. However, recent studies indicate that at least some recent insertions indeed play a functional role in various aspects of the cell. L1-mediated retrotransposition events can be mutagenic, and germline retrotransposition events within the exons or introns of genes can result in null or hypomorphic expression alleles, leading to sporadic cases of human disease ${ }^{10}$. In addition, Lubelsky and Ulitsky demonstrated that sequences enriched in Alu repeats can drive nuclear localization of long RNAs in human cells ${ }^{11}$. An SVA element insertion was recently reported in an intron of TAF1 that ablated expression through aberrant splicing, and is a driving mutation in X-linked Dystonia-Parkinsonism ${ }^{12}$. Another study showed that a recurrent intronic deletion results in the exonization of an Alu element that is found in $6 \%$ of families with mild hemophilia A in France ${ }^{13}$. Somatic L1 retrotransposition can occur in neuronal progenitor cells ${ }^{14-18}$, indicating a possible role for L1s in the etiology of neuropsychiatric diseases ${ }^{19}$. In addition, a mutagenic L1 insertion that disrupted the 16th exon of the APC gene has been shown to instigate colorectal tumor development ${ }^{9}$. Beyond a widespread repertoire of disease associations, mobile elements also influence large scale genome structure. Recent work has demonstrated that transposition events are associated with three dimensional genome organization and the evolution of chromatin structure in human and mouse ${ }^{20-22}$.

A tremendous effort has been made to understand the varied functional outcomes of active MEls. Similar efforts are underway to capture and resolve MEls to discover additional avenues of genetic pathogenesis ${ }^{8,23-28}$. While transformative, these studies were confounded by the shortcomings of existing sequencing methodologies and bioinformatics pipelines, and limited in their ability to access a large ( $\sim 50 \%)$, highly repetitive proportion of the genome ${ }^{29}$. The difficulty in uniquely aligning short-read sequences to repetitive genomic regions likely leads to an under-representation of MEls that have inserted within these regions. Several tools that have been developed to identify non-reference MEls from whole genome short-read data, including the Mobile Element Locator Tool (MELT) ${ }^{27}$, Mobster $^{30}$, Tangram ${ }^{31}$, TEA $^{32}$, and others, are further restricted by the short read length and repetitive nature of mobile elements when resolving longer, non-reference insertions, such as L1Hs and SVA ${ }^{29,33}$. Experimental approaches ranging 
from paired-end fosmid sequencing ${ }^{8,34}$ to PCR capture-based approaches ${ }^{23,35-37}$ have also been developed to capture MEls. However, these methods are intrinsically low throughput and short-read dependent, and they too are hindered by the aforementioned disadvantages.

The advent of long-read sequencing technologies provides a powerful tool for characterizing repeat-rich genomic regions by providing substantially longer sequence reads compared to traditional short-read platforms ${ }^{38,39}$. We have recently applied these technologies to demonstrate that there are at least 2-fold more polymorphic L1Hs sequences in human populations than previously thought ${ }^{29}$. Several existing tools and pipelines have the ability to resolve reference and non-reference MEls in the human genomes; however, most require a whole-genome pipeline for haplotype assembly, local assembly, or cross-platform support ${ }^{40-43}$. This often necessitates whole genome long-read sequencing, which is currently cost-prohibitive at scale and precludes an in-depth exploration into the impact of MEls on human biology and disease. One solution to these barriers is the application of Cas 9 targeted sequence capture with long read sequencing that allows for alignment to unique flanking genomic regions ${ }^{44}$. This approach significantly lowers costs and enables a focused and efficient computational analysis for MEl discovery. Here, we demonstrate the utility of an in vitro Cas 9 enrichment of targeted sequence elements combined with Oxford Nanopore long-read sequencing and established computational methodologies to identify a set of MEls (L1s, AluYs, and SVAs) ${ }^{44}$ that account for over $80 \%$ of currently active mobile elements in the human genome ${ }^{45-47}$. This technology has been previously utilized to resolve a variety of genomic structural variants, including diseases associated with polynucleotide repeats and oncogenic translocation events ${ }^{44,48-50}$. By targeting the Cas9 to subfamily-specific sequences within each element, we demonstrate enrichment of mobile elements across the genome that are both annotated and unannotated in the GRCh38 reference (reference and non-reference MEls, respectively).

\section{Results}

\section{Cas9 targeted enrichment strategy for mobile elements using nanopore sequencing}

We chose GM12878 (NA12878), a member of the CEPH pedigree number 1463 (GM12878, GM12891, GM12892) $)^{51}$, as the benchmark genome in this study. GM12878 is one of the most thoroughly investigated human genetic control samples and has been used in many large-scale genomic projects, such as HapMap ${ }^{52}, 1000$ Genomes Project $^{34,53,54}$, the Human Genome Structural Variation Consortium ${ }^{33,40}$, Genome In A Bottle $e^{55,56}$, and reference genome improvement projects ${ }^{57}$. To precisely capture MEls of interest, we applied Cas 9 targeted nanopore sequencing ${ }^{44,48-50}$ to enrich for five active subfamilies of MEls (L1Hs, AluYb, AluYa, SVA_F, and SVA_E) in GM12878 (Fig.1) as well as L1Hs in the corresponding parental samples. 
a

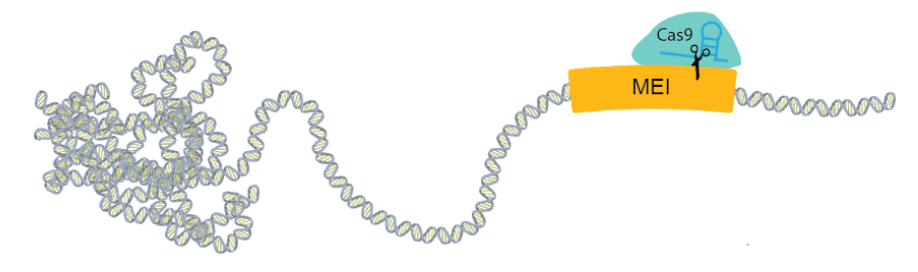

c

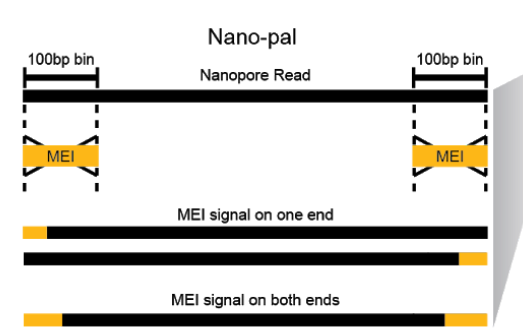

d b

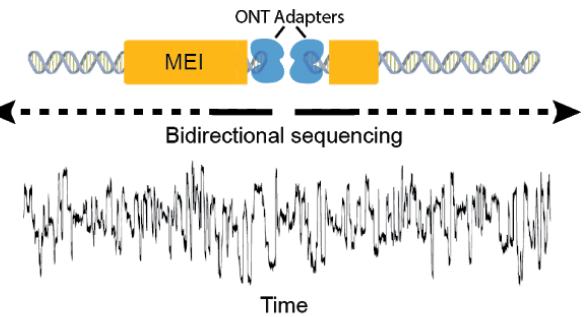

Time

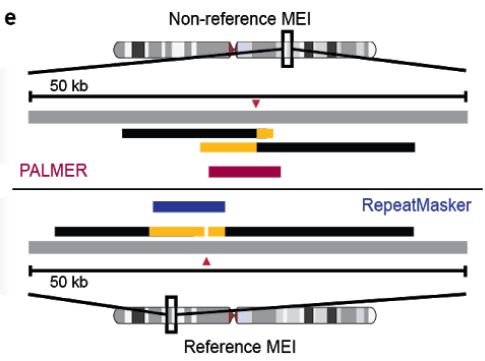

Fig.1: A schematic Cas9 targeted enrichment and Nano-Pal pipeline for mobile elements using nanopore sequencing.

a, Purified genomic DNA (gDNA) is isolated by salting out and then extensively dephosphorylated. Dephosphorylated gDNA is incubated with the Cas9 ribonucleoprotein which is targeted to MEI subfamily-specific sequences near the 3' end of the element. Taq polymerase (not shown), and dATPs (not shown) monoadenylate DNA ends. b, Cas9 cleaved sites are ligated with Oxford Nanopore Technologies (ONT) sequencing adapters and sequenced on a flow cell. Sequencing is bi-directional from the cleavage site. c, Nano-Pal scans the nanopore sequencing reads (black bars) after Cas 9 enrichment for MEI signal on one or both ends. The yellow bar represents MEI consensus sequence or MEI signals in pairwise comparison of Nano-Pal. d, All reads with or without annotated MEl signal are imported into the downstream pipeline. Alignment, classification, and clustering processes are sequentially conducted. Nano-Pal identifies reference and non-reference MEls followed by the inspection of nanopore-specific non-reference MEls (see Methods). e, Examples illustrating capture and alignment of reads containing non-reference L1Hs signal (top) and reference L1Hs signal (bottom). Aligned reads display a non-reference insertion (top) with L1Hs signal (yellow bar) and flanking genomic sequence (black bar). MEI components of reads in non-reference insertions are displayed as overlapping (soft clipping) due to lack of reference genome MEI annotation (grey bar). Aligned reads display annotated reference L1Hs (bottom, yellow bar), flanked by surrounding genomic sequence (black bar), separated by the Cas 9 cleavage site (red triangle). PALMER and RepeatMasker tracks are illustrated in red and blue, respectively.

We designed guide RNAs using unique subfamily-specific sequences within each element to maximize the specificity of Cas9 targeting (Fig. 2, see Methods). Using this approach, we generated a list of candidate $20 \mathrm{bp}$ guide RNAs for each MEI category (Fig.2a-c). We selected an L1Hs candidate guide RNA with the 'ACA' motif at 5929bp of the L1Hs consensus sequence, which distinguishes the L1Hs subfamily from other L1 subfamilies (e.g. L1PA) ${ }^{23,37,47}$. For AluY and SVA, 18 unique guide RNA candidates were obtained (one for AluYb, three for AluYa, seven for SVA_F, and six for SVA_E) (see Methods, Supplementary Fig.1,2 and Supplementary Table 1). Candidates were further prioritized to those with the largest number of subfamily-specific bases and proximity to the 3' end of the MEI sequence, near the polyA tail, which is an obligate component of the TPRT mechanism of retrotransposition ${ }^{58,59}$. From the pool of candidates, a single guide RNA for each MEI subfamily was selected for downstream 
enrichment experiments (Supplementary Table 1). After Cas9 enrichment and read processing, we assessed the cleavage sites of all five guide RNAs (Fig. 2d and Supplementary Fig. 3). The resulting distribution showed a vast majority of the forward-strand reads start at the third or fourth base-distance from the 'NGG' PAM site, and reverse-strand reads begin at the seventh base. This is consistent with previous characterization studies of Cas9 cleavage activity ${ }^{60}$. Furthermore, we observed strand bias with approximately 4.6-fold more reads on the forward strand compared to the reverse strand, which has been hypothesized to be caused by Cas9 remaining bound after cutting and obstructing adapter ligation and sequencing ${ }^{44,61}$. We detected directional sequencing biases within different MEI subfamilies and enrichment runs (Supplementary Table 2, Supplementary Fig.3).
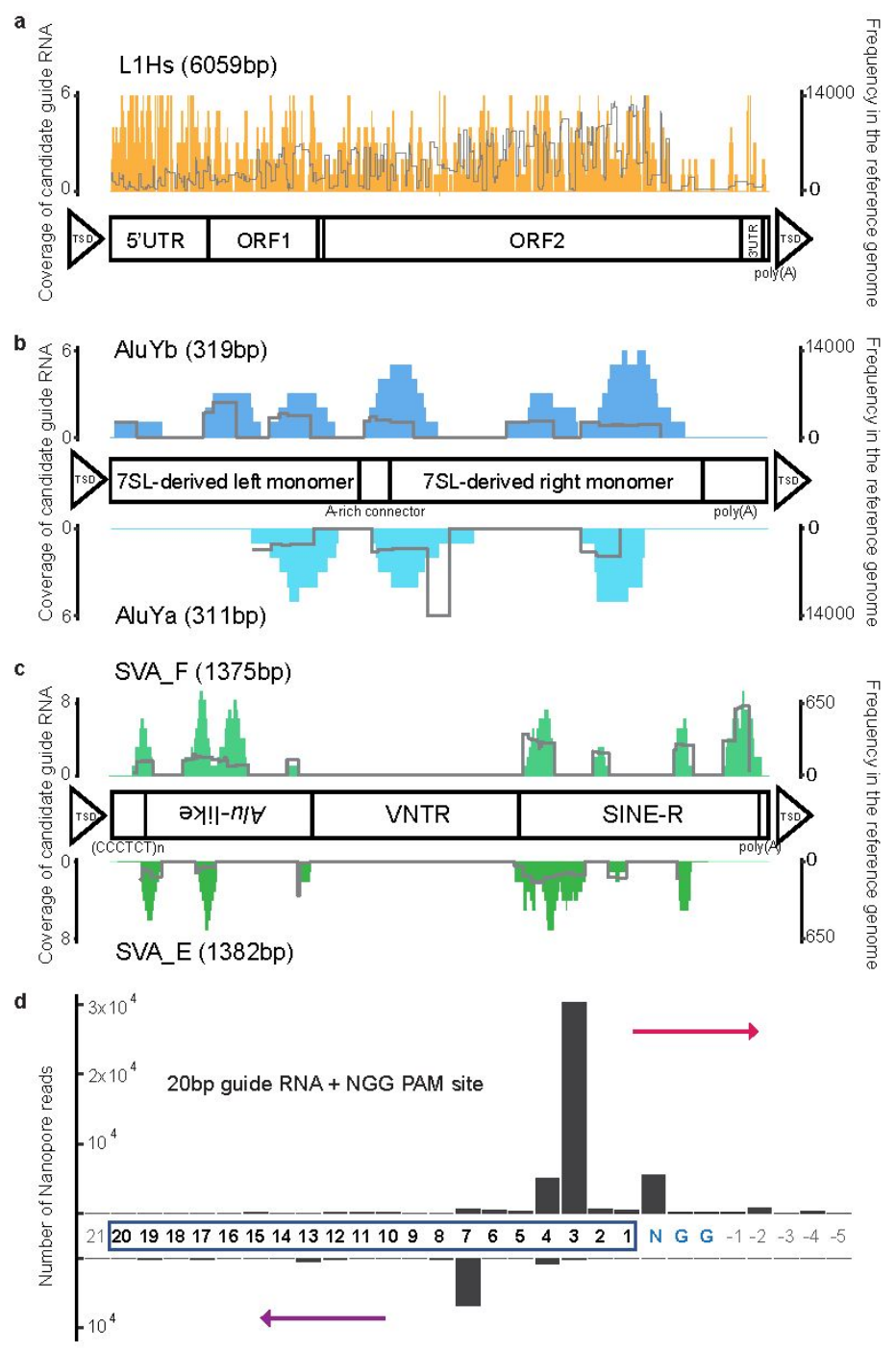

Fig.2: Guide RNA design for MEls and guide RNA cleavage-site distribution.

a, Distributions of candidate guide RNAs (left Y-axis) in the L1Hs consensus sequence and structure information. The right $\mathrm{Y}$-axis indicates frequency of corresponding candidates in the reference genome sequence. b, Upper panel shows the distribution for AluYb and the lower panel for AluYa. c, Upper panel 
shows the distribution for SVA_F and the lower panel for SVA_E. d, Cleavage-site distribution of all guide RNAs in this project. The $x$-axis indicates the position where the read ends or begins, with the number depicting the base distance from the PAM site (NGG). The PAM site (NGG) is colored blue and guide RNA bases are highlighted by a rectangle. Bases outside of the guide RNA or the PAM site are colored grey. The $y$-axis is the number of nanopore reads counted. The upper bar represents reads with forward strand sequencing outward from the 3' end of the guide RNA (red arrow) and the lower bar represents reads with reverse strand sequencing outward from the 5' end of the guide RNA (purple arrow).

We developed a computational pipeline, Nano-Pal, to analyze captured long reads after base-calling and trimming, estimate the on-target rate of Cas 9 enrichment from MEI signals on the ends of reads, and identify reference and non-reference MEls (Fig.1b,c). Due to the frequency of targeted MEls in the genome, an individual nanopore read may harbor a MEl signal on one or both ends. Reads with a single-end MEI signal had similar read lengths within all MEI experiments, yet were significantly larger than reads with two-end MEI signals. This was especially true in L1Hs experiments (L1Hs 1.9-fold, AluY 1.1-fold, SVA 1.4-fold, Supplementary Fig.4). To better distinguish non-reference MEI signals from those present in the reference, particularly where non-reference MEls are embedded into reference $\mathrm{MEIs}^{29}$, the pre-masking module from an enhanced version of our long-read non-reference MEI caller, PALMER ${ }^{29}$, was implemented into Nano-Pal (Fig.1d). This enabled identification and masking of reference MEls in individual long-reads, enhancing detection of non-reference MEI signals within the remaining unmasked portion ${ }^{29}$. Non-reference and reference MEls were then summarized by clustering nearby nanopore reads.

Cas9 targeted enrichment efficiently captures mobile element signals in nanopore reads

We performed a total of seven distinct MEl enrichment experiments across two types of nanopore sequencing platforms: Oxford Nanopore Flongles and MinIONs. Five of the experiments, one for each MEI subfamily, were sequenced on Flongle flow cells. One MinION flow cell was used to sequence an L1Hs enrichment, and another MinION flow cell was used to sequence a pooled sample of all five MEls (see Methods) (Table 1 and Supplementary Table 3). The N50 ranged from $14.9 \mathrm{~kb}$ to $32.3 \mathrm{~kb}$ in all the experiments after base calling and quality controls.

To estimate the enrichment efficiency for different flow cells and MEI subfamilies, all passed reads were classified into three categories: on-target, close-target, and off-target (see Methods). The on-target rate for nanopore reads from a single $\mathrm{L} 1 \mathrm{Hs}$ experiment on a Flongle flow cell, including both reference and non-reference MEls, was 56.9\%. Relatively lower on-target rates were observed for the other MEls on the Flongle flow cell: $46.7 \%$ for AluYb, $23.8 \%$ for AluYa, 5.8\% for SVA_F, and 2.3\% for SVA_E (Table 1). When an L1Hs enrichment experiment was sequenced on a MinION flow cell, the on-target rate was approximately $35.0 \%$ (FAL11389) and 23.3\% for a pooled MEI run (FAO84736). Compared to earlier studies (2.09\% in Flongle and $4.61 \%$ in MinION $)^{44}$, these results show substantially improved enrichment, with a 1- to 25-fold increase relative to the Flongle flow cell, and over 5-fold enrichment relative to the MinION flow cell. These enrichment increases are likely due to the frequency of the targets in the genome. Overall, our approach reaches an average of $44 \%$ of nanopore sequencing reads with target MEI signal from these seven flow cell runs. 
Table 1 Efficient enrichment of mobile element signals in nanopore reads.

Summary of seven representative flow cells: five individual Flongle flow cells for L1Hs (ABB607), AluYb (ACK645), AluYa (ACK655), SVA_F (ACK629), and SVA_E (ABO395) each, one individual MinION flow cell for L1Hs (FAL11389), and one pooled MinION flow cell for five MEls (FAO84736).

\begin{tabular}{|c|c|c|c|c|c|c|c|}
\hline \multirow{2}{*}{ MEI } & \multirow{2}{*}{ Run } & \multirow{2}{*}{ Flow cell } & \multirow{2}{*}{ Read number } & \multicolumn{2}{|c|}{ On-target } & \multirow{2}{*}{$\begin{array}{c}\text { Close-target } \\
\text { Reference } \\
\end{array}$} & \multirow[t]{2}{*}{ Off-targe } \\
\hline & & & & Reference & Non-reference & & \\
\hline \multicolumn{8}{|c|}{ Individual } \\
\hline L1Hs & ABB607 & \multirow{5}{*}{ Flongle } & 4,102 & $49.6 \%$ & $7.3 \%$ & $16.8 \%$ & $26.3 \%$ \\
\hline AluYb & ACK645 & & 2,271 & $40.2 \%$ & $6.5 \%$ & $1.0 \%$ & $52.2 \%$ \\
\hline AluYa & ACK655 & & 12,513 & $18.0 \%$ & $5.8 \%$ & $10.3 \%$ & $65.9 \%$ \\
\hline SVA_F & ACK629 & & 14,106 & $3.7 \%$ & $2.1 \%$ & $3.7 \%$ & $90.6 \%$ \\
\hline SVA_E & ABO395 & & 7,297 & $1.7 \%$ & $0.6 \%$ & $0.2 \%$ & $97.5 \%$ \\
\hline L1Hs & FAL11389 & MinION & 110,029 & $30.7 \%$ & $4.3 \%$ & $37.9 \%$ & $27.1 \%$ \\
\hline Pooled & FAO84736 & MinION & 105,410 & $20.1 \%$ & $3.2 \%$ & $38.4 \%$ & $38.3 \%$ \\
\hline L1Hs & & & & $8.9 \%$ & $1.6 \%$ & $33.9 \%$ & \\
\hline AluYb & & & & $7.0 \%$ & $12 \%$ & $32 \%$ & \\
\hline AluYa & & & & $2.8 \%$ & & & \\
\hline SVA_F & & & & $1.2 \%$ & $04 \%$ & $13 \%$ & \\
\hline SVA_E & & & & $0.2 \%$ & $0.4 \%$ & $1.3 \%$ & \\
\hline
\end{tabular}

To further assess the improved enrichment of MEI subfamilies, the extent of representation of MEl targets with high sequence identity were examined within the data. A portion of the enrichment data contained 'close-target' reads that resemble related subfamilies of the intended targets and can be explained by the base mismatch tolerance between the guide RNA sequence and the targeted $\mathrm{MEI}$ sequence ${ }^{62,63}$. For $\mathrm{L} 1 \mathrm{Hs}$, a rate of $16.8 \%$ on the Flongle and $33.9 \%$ to $37.9 \%$ on the MinION flow cell was observed, with close-target reads mapping to reference L1PA regions. Flongle sequencing of AluYa had a rate of $10.3 \%$ of close-target reads to other reference AluY elements, in contrast to AluYb where a dramatically reduced 'close-target' rate of $1.0 \%$ was observed (Table 1). This enhanced specificity may be explained by a specific insertion sequence within AluYb (5'-CAGTCCG-3') that was included in the guide RNA and is unique to the youngest $A l u$ elements $(A l u Y b)^{46}$ of the genome. For the SVA Flongle sequencing, 'close-target' rates of $3.7 \%$ and $0.2 \%$ to the other reference SVAs were observed in the SVA_F and SVA_E enrichment, respectively.

Cas9 enrichment and nanopore sequencing rapidly saturates reference and non-reference MEls

Due to the possibility that the guide RNA may bind to off-target sites and direct Cas9 to cut in MEls that are not perfectly matched to the guide sequence ${ }^{62,63}$, we established expectation thresholds to evaluate the number of captured reference and non-reference MEls. The reference $\mathrm{MEI}$ sets were obtained from RepeatMasker ${ }^{64}$. A 'PacBio-MEl' callset in GM12878 was generated by comprising a mapping-based callset and an assembly-based callset as a comprehensive gold standard set for non-reference MEls (see Methods). The PacBio-MEI includes 215 L1Hs, 362 AluYb and 593 AluYa in 1404 Alus, and 33 SVA_F and 24 SVA_E in 72 
SVAs (Supplementary Fig.5 and Supplementary Table 4). Three categories (lower, intermediate, and upper) were defined that contain a number of reference and non-reference MEls from each subfamily (Supplementary Table 5). Each threshold classifies MEls depending on the extent of allowed mismatches between the guide and the sequence. The lower-bound is the most stringent and requires a perfect match between the guide sequence and the MEI. The intermediate-bound is less stringent and can tolerate three or fewer mismatches. We consider this to be the closest estimation to the number of MEls that a guide RNA could reasonably capture among these three boundaries. Finally, the upper-bound is the least stringent and most inflated, requiring that at least $60 \%$ of the guide sequence matches the MEI (see Methods).
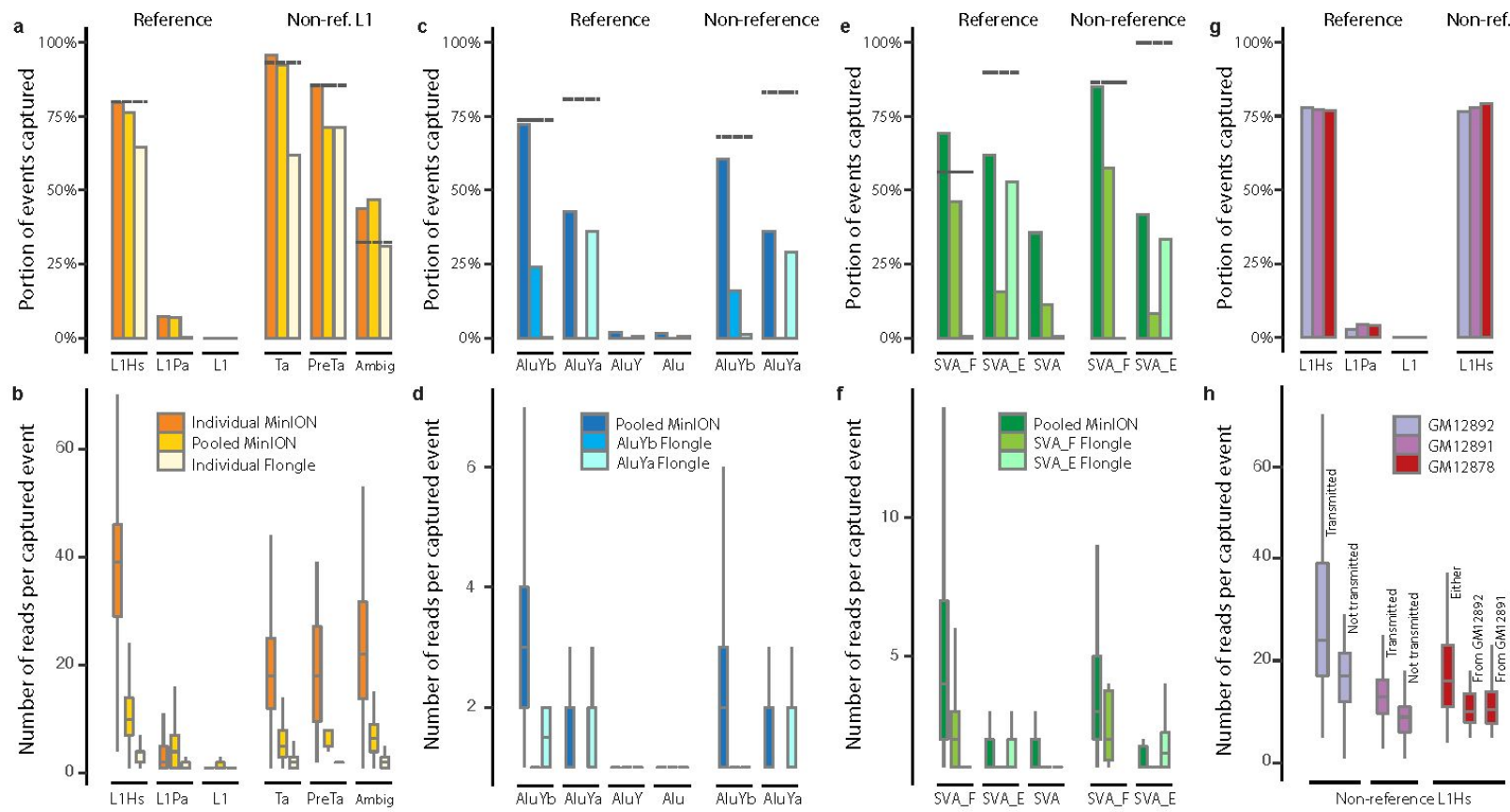

Fig.3: Systematic evaluation of known MEls captured by nanopore Cas9 enrichment approach in different flow cells.

a, Known L1Hs in GM12878 recovered by Cas9 targeted enrichment from the individual MinION flow cell (FAL11389), pooled-MEI MinION flow cell (FAO84736), and individual Flongle flow cell (ABB607), displayed as a proportion of the upper-bound known reference L1Hs, L1Pa, and other L1 as well as non-reference (non-ref.) L1Hs from the PacBio-MEI set. Non-reference L1Hs were divided into different subfamilies (L1Ta, L1PreTa, and L1Hs with ambiguous subfamilies). Dotted-grey line represents the intermediate values (as proportion) of MEls that the guide RNA binds when allowing a $\leq 3 \mathrm{bp}$ mismatch or gap. b, Number of supporting reads of each captured L1 in the context of a. c, Known AluY elements in GM12878 recovered by Cas9 enrichment on one pooled MinION flow cell (FAO84736), one individual AluYb Flongle flow cell (ACK645), and one individual AluYa Flongle flow cell (ACK655). d, The number of supporting reads of each captured Alu element in the context of c. e, Known SVA elements in GM12878 recovered by Cas9 enrichment on one pooled MinION flow cell (FAO84736), one individual SVA_F Flongle flow cell (ACK629), and one individual SVA_E Flongle flow cell (ACK395). f, The number of supporting reads of each captured SVA element in the context of $\mathbf{e}$. g, Known L1Hs captured in the GM12878 trio by Cas9 enrichment on one pooled MinION flow cell (FAL15177). $\mathbf{h}$, The number of supporting reads of each captured non-reference L1Hs based on transmission in the GM12878 trio. The non-reference L1Hs in the parents (GM12892 and GM12891) were categorized into transmitted and 
not-transmitted. The non-reference L1Hs in the child (GM12878) were categorized as insertions inherited from GM12892 o r GM12891, and from either parents (unknown parental lineage). In b, d, f, h, error bars range from $\mathrm{Q} 1-1.5 \mathrm{IQR}$ to $\mathrm{Q} 3+1.5 \mathrm{IQR}$ (IQR, interquartile range) and outliers are not shown.

Upon comparing our MEI enrichment data to the aforementioned intermediate value estimates (Fig.3a,b, Supplementary Fig.6, and Supplementary Table 5), the individual and the pooled MinION flow cell captured 100\% (35.8 mean coverage) and 96.0\% (10.5 mean coverage) of known reference $\mathrm{L} 1 \mathrm{Hs}$ with on-target reads, respectively. The individual Flongle captured $81.2 \%$ (2.7 reads mean coverage) of known reference L1Hs. For the non-reference L1Hs, the individual and pooled MinION flow cell captured $100 \%$ and $99.4 \%$ for all the L1Hs subfamilies, respectively. The individual Flongle captured $66.4 \%, 83.3 \%$, and $95.2 \%$ for non-reference L1Ta, L1PreTa, and L1Ambig, respectively. Our results showed that only one of the MinION flow cells (FAL11389 or FAO84736) was necessary to capture most of the known reference and estimated non-reference L1Hs subfamilies in the genome when considering intermediate values, indicating a very high sensitivity of guide RNA targeting in the experiments.

Compared to the least stringent upper-bound estimates, $64.5 \%$ and $79.7 \%$ of known reference L1Hs were captured using the individual Flongle and MinION flow cell, respectively. Non-reference L1Hs capture ranged from $53.0 \%$ to $80.0 \%$, compared to $4.1 \%$ to $7.3 \%$ of close-target L1PA elements, and less than $0.01 \%$ of off-targeting to other L1 elements (Fig.3a,b, Supplementary Fig.6, and Supplementary Table 5). The high percentage of elements captured that were on-target versus the other categories, including off-target, indicates the high specificity of the guide RNA to L1Hs in the enrichment. The read depth of the reference and non-reference $\mathrm{L} 1 \mathrm{Hs}$ elements observed in these MinION flow cells has an approximate ratio of 2:1 (Fig.3b), consistent with the expectation that reference MEls are homozygous, and a considerable portion of non-reference MEls are heterozygous ${ }^{65}$.

For AluY subfamilies, individual Flongles were utilized for separate runs of AluYb (ACK645) and AluYa (ACK655), and one pooled MinION flow cell (FAO84736) (Fig.3c,d, Supplementary Fig.6, and Supplementary Table 5). 93.9\% (3.5 mean coverage) reference and $88.0 \%$ (2.4 mean coverage) non-reference AluYbs were captured from the pooled MinION flow cell run, based on intermediate values. Similar to the L1Hs, the MinION flow cell was able to capture a vast majority of $A l u Y b$ elements when considering intermediate values, indicating high sensitivity performance of the AluYb enrichment. A relatively lower rate of capture for reference (52.7\%, 1.6 mean coverage) and non-reference (42.0\%, 1.4 mean coverage) AluYa enrichment was observed in the pooled MinION flow cell run based on intermediate values. Cross-capture rate from the two individual Flongle flow cells was less than $0.1 \%$, and close- and off-target reference elements were $<0.1 \%$ and $2 \%$ for the Flongles and MinION, respectively, indicating a high specificity of the guide RNA for each AluY subfamily (Fig.3c and Supplementary Table 5).

Similar enrichment rates were obtained from the two individual Flongle flow cells for SVA_F (ACK629) and SVA_E (ACK395), and in the pooled MinION flow cell (FAO84736) (Fig.3e,f, Supplementary Fig.6, and Supplementary Table 5). 100\% (4.6 mean coverage) reference and $96.6 \%$ (3.9 mean coverage) non-reference SVA_F were captured from the pooled MinION flow cell run based on intermediate values. A relatively lower rate of capture for reference $(68.7 \%, 1.9$ mean coverage) and non-reference $(41.7 \%, 1.5$ mean coverage) SVA_E 
enrichment was observed in the pooled MinION flow cell run based on intermediate values. The close-target reference SVAs capture rate was relatively high in two of the runs $(35.6 \%$ in MinION flow cell and $11.4 \%$ in ACK629 Flongle flow cell). This could be due to SVAs sharing less base pair substitutions among their subfamilies compared to the other MEl families, as it is the youngest retrotransposon family found in the hominid lineage ${ }^{45}$.

Our results indicate that an individual MinION flow cell (FAL11389) is able to completely (100\%) capture reference and non-reference instances of a single MEI subfamily (L1Hs) compared to sequencing on the smaller Flongle flow cells. More importantly, a pooled run of an unbarcoded, five MEI subfamily enrichment experiment can recover the vast majority of known reference and non-reference MEls (96.5\% L1Hs, 93.3\% AluYb, 51.4\% AluYa, 99.6\% SVA_F, and 64.5\% SVA_E) in the genome when considering elements with a $\leq 3 \mathrm{bp}$ mismatch to the guide RNA. Such an approach outperformed individual Flongle flow cells and approached the same capture level as the single MEI subfamily MinION run (Fig.3, Supplementary Fig.6, and Supplementary Table 3,5). This suggests that the MinION flow cell has ample sequencing capacity to accommodate each experiment with negligible competition between samples, despite sequencing multiple $\mathrm{MEI}$ enrichments on one platform. Finally, a substantial fraction of reference and non-reference $\mathrm{MEI}$ events can be captured in a single MinION sequencing experiment with multiple supporting reads. With further optimization of the enrichment and sequencing methodologies, it is plausible to fully saturate reference and non-reference MEls in a single experimental iteration.

\section{Detectable transmission of non-reference L1Hs within a trio}

To trace the transmission of non-reference L1Hs in GM12878 from the parents, another enrichment experiment of L1Hs elements was performed in GM12878, GM12891, and GM12892 (Fig.3g,h and Supplementary Table 3). The on-target rate for L1Hs ranged from $34.3 \%$ to $40.4 \%$ in the individual Flongles for GM12891 and GM12892 (parents), which mirrors the on-target rate of GM12878 (child) (Supplementary Table 6).

Transmission of non-reference L1Hs to GM12878 from the parental genomes was further examined using available GM12891 and GM12892 sequencing data. As the parental genomes lack sufficient long-read sequencing data, we utilized MELT to resolve non-reference L1Hs callsets from high-coverage Illumina short-read sequencing data. This analysis yielded 123 and 118 high confidence, non-reference L1Hs in GM12892 and GM12891, respectively. The number of MEls identified in the MELT call sets are relatively lower than the number $(n=205)$ detected in the 'PacBio-MEl' set for GM12878, consistent with previous observations that long-reads are more sensitive for MEI discovery ${ }^{29,33,40}$ (Supplementary Table 4). Additional evidence of transmission can be derived by the enrichment of reads from non-reference MEls in GM12878. We expect MEls that can be transmitted from either parent will have higher read coverage due to a portion of these being homozygous, and single parent transmitted MEls will be heterozygous in GM12878. As predicted, an enrichment of approximately 1.52-fold (17.6 vs 11.6 mean coverage) was observed for these reads (Fig.3h). Similarly, the 'not transmitted to child' non-reference L1Hs in parent samples should be heterozygous and were observed to be depleted by approximately $0.56 \sim 0.72$-fold (17.4 vs. 31.3 mean coverage in GM12892 and 10.7 
vs. 14.8 mean coverage in GM12891) of the nanopore reads that have been transmitted to the child (Fig.3h). These observations showed an expected supporting-read distribution of non-reference $\mathrm{L} 1 \mathrm{Hs}$, supporting the efficient nanopore Cas9 targeted enrichment in the pooled trio samples.
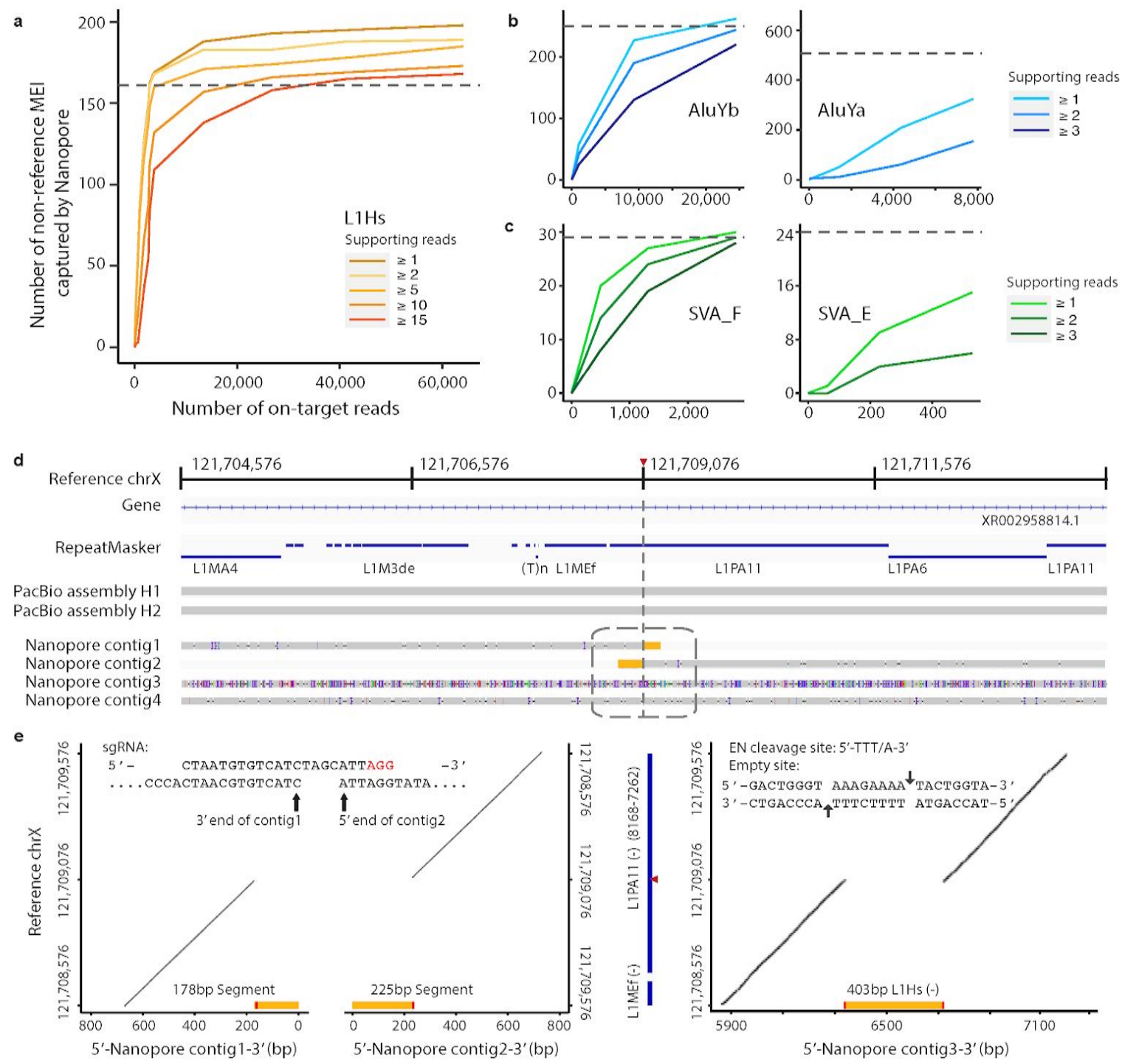

Fig.4. Non-reference MEls captured by nanopore Cas9 enrichment approach.

a, Number of non-reference L1Hs captured by nanopore Cas9 enrichment at different on-target read coverages for different supporting read cutoffs. The dotted-grey line represents the theoretical number of MEls that the guide RNA binds when allowing a $\leq 3 b p$ mismatch or gap in the PacBio-MEl set $\mathbf{b}, \mathbf{c}$, Number of non-reference AluYb, AluYa, SVA_F, and SVA_E, respectively, captured by nanopore Cas9 enrichment at different on-target read coverages. Axis labels and theoretic guide number as in a. d, An example of non-reference L1Hs specifically captured by nanopore sequencing at chrX:121,709,076. The tracks from top to bottom are as follows: reference coordinates with a red triangle represent the insertion site, gene track, RepeatMasker track (blue bars) with reference element annotation, PacBio contigs 
assembly for two haplotypes, four nanopore local-assembled contigs by CANU from different classifications of nanopore reads based on insertion signals (contig1, signal on 3' end; contig2, signal on 5 ' end; contig3, signal in the middle of the read; and contig4, no signal). e, Recurrence (dot) plots for nanopore contigs versus the reference region chrX:121,708,576-121,7089,576 sequence. Left panel shows the most 3 ' end of contig1 and the most 5' end of contig2 versus the reference sequence. Yellow bar represents the non-reference L1Hs sequence contained in the contig. The red bar represents one side of the target site duplication motif for the non-reference L1Hs contained in the contig. The upper part of this panel demonstrates sequences at the end of two contigs regarding the cleavage site when aligning to the guide RNA sequence. Blue bars in the middle panel represent the RepeatMasker track with reference L1 information annotated and the red triangle represents the insertion site in the reference L1 region. The right panel shows contig3 versus the reference sequence. Details of this non-reference L1Hs are detailed in the panel, including length, strand, empty site, and endonuclease (EN) cleavage site sequence.

Cas9 enrichment and nanopore sequencing captures non-reference mobile elements in complex genomic regions

To estimate the efficacy of enrichment for non-reference MEls with different sequencing coverage, we manually inspected each non-reference MEI reported by Nano-Pal and performed subsequent saturation analysis for all flow cells (Fig.4, see Methods). We find few additional L1Hs insertions by including additional on-target reads beyond approximately 30,000, using a cutoff of 15 supporting reads (Fig.4a). This is consistent with the observation that the MinION (individual or pooled, usually with $>100 \mathrm{k}$ passed reads) has the ability to capture most non-reference L1Hs. In addition, there was no observable enrichment bias of MEl subfamilies from different flow cells (Supplementary Fig.7).

We examined the 182 non-reference L1Hs in GM12878 that overlapped with the PacBio-MEI set. Of these $175(96.2 \%)$ could be accounted for by the parental (GM12891 and GM12892) sequencing data (Supplementary Fig.8), and three overlapped known polymorphic insertions ${ }^{40}$. The remaining four non-reference $\mathrm{L} 1 \mathrm{Hs}$ are located within centromeric regions, which could be missed in the parental samples due to lack of supporting reads. In addition, we observed 601 non-reference AluY (including 323 AluYb and 263 AluYa) and 49 non-reference SVA (including 30 SVA_F and 15 SVA_E) that overlapped with the PacBio-MEl set. We further examined the set of MEls that were captured exclusively by Cas 9 targeted enrichment and nanopore sequencing, but not found in the PacBio-MEI intersection. We identified 16 additional L1Hs insertions as nanopore specific with $\geq 4$ supporting reads that had been missed by the PacBio-MEl set. In addition, we detected 5 AluY elements that were specifically captured by nanopore reads in the GM12878 genome. After refinement and inspection, we generated a full set of non-reference MEIs (198 L1Hs, 606 AluY, and 49 SVA) captured by Cas9 enrichment and nanopore sequencing in the GM12878 genome. Of note, all intermediate-value calls of PacBio-MEl for L1Hs, AluYb, and SVA_F were recovered in this study (Supplementary Table $5,7,8)$.

One non-reference L1Hs insertion at chrX:121,709,076 was particularly intriguing. The PacBio genome assembly-based approach overlooked this insertion, as it fell within a 'reference L1 rich' region (Fig.4d). Upon further inspection, this event was supported as a 403bp heterozygous 
L1Hs insertion by the existence of significant retrotransposition hallmarks, as well as recurrence (dot) plots $^{29,66}$ (Fig.4e, see Methods). This insertion also shares a high sequence identity with a nearby reference L1PA11 element (Supplementary Table 7). The decrease in efficacy of the PacBio assembly-based approach in this region could be explained by the intricate nested ' $L 1$ in L1' structure and observed heterozygosity (Fig.4d). Likewise, the AluY nanopore Cas9 enrichments captured interesting non-reference AluY instances: A homozygous AluYb8 insertion at chr19:52384635, an exonic region within the ZNF880 gene, was reported to alter RNA expression due to the Alu element's effects on the RNA secondary structure ${ }^{67}$. Another heterozygous AluYa5 insertion at chr16:69157709 was located within a reference AluJr region, indicating a potential nested 'Alu in $A / u$ ' structure that could hinder non-reference AluY discovery. These observations demonstrate the high sensitivity of nanopore Cas9 enrichment, suggesting its feasibility for MEI discovery in complex genomic regions.

\section{Discussion}

Here we describe our design and implementation of Cas9 targeted nanopore sequencing to enrich for retrotransposition competent, repetitive mobile elements in the human genome ${ }^{44}$. After carefully designing guide RNAs to each MEI subfamily and coupling the enrichment with an established computational pipeline, our approach reaches an average of $44 \%$ nanopore sequencing reads with target $\mathrm{MEI}$ signals. We recovered a vast majority of reference and known non-reference mobile elements (96.5\% L1Hs, 93.3\% AluYb, 51.4\% AluYa, 99.6\% SVA_F, and $64.5 \%$ SVA_E) in the genome using only a single MinION flow cell. In addition, we discovered 21 non-reference MEls within the GM12878 genome that were previously missed by other orthogonal long-read pipelines. Our data suggest that a MinION flow cell is ideal for a pooled, multiple-element enrichment experiment, as a prohibitively reduced enrichment or extensive cross-capturing of subfamilies was not observed. The work presented here highlights the potential of targeted enrichment and nanopore sequencing to rapidly discover distinct MEls, and cements an experimental foundation to probe even the most elusive mobile element insertion events.

Cas9 targeted enrichment paired with nanopore sequencing has the potential for resolving complex structural variation, previously obfuscated by sequencing and computational limitations. We leveraged the nanopore Cas9 targeted sequencing ${ }^{44}$ method to target active retrotransposons in the human genome in a discovery-based approach. To our knowledge, this is the first application of this method for repetitive mobile element detection. Our experiments indicate that by utilizing guides targeted to specific subfamily sequences, both reference and non-reference insertions can be efficiently enriched and mapped with multiple supporting reads on even the smallest of nanopore sequencing flow cells. Moreover, we demonstrate that individual sequencing experiments readily capture a majority of reference and non-reference elements. In both pooled and single element experiments, MEls of five subfamilies are robustly enriched, suggesting that this method is widely applicable across mobile elements, and most suitable for high copy genomic elements. Guide RNA design process is straightforward, and targeting elements based on subfamily nucleotide differences captures both reference and non-reference elements, with negligible loss of sequencing to related close subfamilies or 
off-targeting. Unlike other Cas9 targeted enrichment experiments, on-target (reference and non-reference) rates for our method are comparatively higher, exceeding $50 \%$ in some cases. While this is likely a consequence of the number of genomic copies of the targeted element and, to a lesser extent, the fidelity of the guide sequence, it reiterates this method is particularly suitable for MEI discovery.

The preponderance of uncharacterized MEls, taken together with their potential contribution to genomic variation and disease, emphasizes the critical need for efficient mobile element detection strategies. Our experiments using Cas9 enrichment and nanopore sequencing can quickly map active mobile elements in the human genome, as well as their larger genomic context. Since Cas 9 enrichment has been used to target rare rearrangement events ${ }^{68}$, implementation of this approach to detect unique, de novo, or somatic mobile element insertions is a feasible endeavor. Identification of particularly rare insertion events, such as mosaicism resulting from retrotransposition in early embryogenesis, is possible. While we surmise that the vast majority of MEls can be captured using this approach, it is important to recognize that some genomic locations may persistently conceal recently transposed elements. Centromeric regions and long palindromic repeats are examples of complex genomic features that could be recalcitrant to $\mathrm{MEI}$ discovery ${ }^{69}$. With $\mathrm{N} 50$ s of more than $25 \mathrm{~kb}$, we observed some MEI signals in centromeric and highly repetitive, palindromic regions from our nanopore sequencing reads. However, these regions still complicate mapping, requiring substantially longer sequencing and comprehensive analysis to confidently pinpoint elusive insertions ${ }^{70}$. Merging the enrichment experiments discussed here with improved commercial kits and extremely high molecular weight genomic DNA, may be critical for preserving the extremely long fragments necessary to map MEls in complex genomic landscapes. The importance of mobile element activity in shaping the genomes they inhabit cannot be overstated. Even beyond the scope of the human genome, mobile element activity plays an intricate role in evolution across many organisms ${ }^{71,72}$. Accelerated discovery of active mobile elements and other repetitive genetic elements will expand our understanding of their contributions to phenotypic diversity in genomes from every form of life.

\section{Methods}

Cell culture, counting, and genomic DNA isolation

The following cell lines/DNA samples were obtained from the NIGMS Human Genetic Cell Repository at the Coriell Institute for Medical Research: GM12878, GM12891, GM12892. Each cell line was cultured at $37^{\circ} \mathrm{C}, 5 \% \mathrm{CO}_{2}$ in RPMI 1640 media (ThermoFisher, 11875093) with L-glutamine, and supplemented with $15 \%$ fetal bovine serum (ThermoFisher, 10437028) and $1 \mathrm{x}$ antimycotic-antibiotic (ThermoFisher, 15240112). Cells were regularly passed and the media replenished every 3 days.

High molecular weight genomic DNA was isolated from GM12878, GM12891, and GM12892 using a "salting out" method ${ }^{73}$ with the following modifications. Lymphoblastoid cells were harvested from culture and centrifuged at $500 \mathrm{xg}$ for 10 minutes at $4^{\circ} \mathrm{C}$. Each pellet was washed 
in $4^{\circ} \mathrm{C} 1 \mathrm{X}$ phosphate buffered saline (PBS) and cell number was counted using the Countess II. Washed cell pellets were resuspended in $3 \mathrm{~mL}$ of nuclei lysis buffer $(10 \mathrm{mM}$ Tris- $\mathrm{HCl} \mathrm{pH} 8.2$, $400 \mathrm{mM} \mathrm{NaCl}$, and $2 \mathrm{mM}$ EDTA pH 8.2). $200 \mathrm{uL}$ of $10 \%$ SDS was added to the resuspension and inverted until evenly mixed. $50 \mathrm{uL}$ of RNase $A(10 \mathrm{mg} / \mathrm{mL})$ was added and the lysate was rotated at $37^{\circ} \mathrm{C}$ for 30 minutes followed by addition of $50 \mathrm{uL}$ of proteinase $\mathrm{K}(10 \mathrm{mg} / \mathrm{mL})$ and rotation at $37^{\circ} \mathrm{C}$ overnight. $1 \mathrm{~mL}$ of saturated $\mathrm{NaCl}$ solution was added to the lysate and mixed by handheld shaking until evenly mixed. The sample was then centrifuged at 4000xg for 15 minutes at room temperature. The supernatant was transferred into a new $1.5 \mathrm{~mL}$ microcentrifuge tube. 2 volumes of $100 \%$ ethanol were added to the supernatant and the tube was inverted approximately 20 times, or until the precipitate coalesced. The precipitate was isolated via spooling with a sterile $\mathrm{p} 10$ pipette tip and resuspend in a sufficient volume of $1 \mathrm{X}$ TE buffer ( 250-500uL, depending on starting amount of cell material). Genomic DNA was passed through a $27 \mathrm{G}$ needle 3 times and stored at $4{ }^{\circ} \mathrm{C}$. The DNA concentration was measured using a Qubit 3 Fluorimeter and the dsDNA Broad Range Assay kit (ThermoFisher, Q32850)

\section{Design of unique guide RNAs for L1Hs, AluYb, AluYb, SVA_F, and SVA_E}

To maximize the enrichment performance for each MEl subfamily, the guide RNA (gRNA) candidates were designed to bind to the unique sequences within each subfamily. A pairwise comparison was conducted for the target MEls with other, non target subfamilies. The consensus sequences for each target MEI subfamily were obtained from Repbase ${ }^{74}$, namely L1Hs in the L1 family, AluYa5 and AluYb8 in the Alu element family representing the AluYa and AluYb subfamily, and SVA_E and SVA_F in the SVA family. These subfamilies account for over $80 \%$ of currently active mobile elements in the human genome ${ }^{45-47}$. The consensus sequences of L1PA2, AluY, primate Alu, and SVA_D were retrieved from Repbase and included as outgroups in the comparison analysis. Furthermore, AluYa5 was added as an outgroup in the design of the gRNA for AluYb, AluYb8 for AluYa, SVA_E for SVA_F, and SVA_F for SVA_E to avoid enrichment across target MEls.

Guide RNA target sites (20bp sgRNA + 3bp NGG PAM site) for S. pyrogenes Cas9 were identified that are within unique MEI regions to obtain optimal guide candidates. Jellyfish2. ${ }^{75}$ was utilized to create a $k$-mer $(\mathrm{k}=23)$ index for the sequences of these unique regions, and 23mers with a 5' 'CC' or 3' 'GG' were selected as gRNA candidates. The frequency of gRNA candidates and the three base substitution options in the 'NGG' PAM site for each candidate in the reference genome was calculated to confirm that the number of unique guide sequences is similar to the genomic reference MEl sequence frequency (Supplementary Table 1 and Supplementary Fig.1,2). The guide RNA candidates of AluY and SVA with unique sequences into were categorized into different tiers: Tier0, sequence has subfamily-specific bases in GG/CC of the PAM site; Tier1, the frequency of 23 mer falls into a reasonable range (<2-fold of target MEI frequency) in reference genome; Tier2, the sequence has only subfamily-specific bases at the $\mathrm{N}$ site of the PAM or the frequency of the 23 mer falls out of a reasonable range. $\mathrm{A}$ gRNA sequence falling in TierO was considered an ideal candidate. 


\section{On-target boundary calculations for MEls}

Using the final gRNA selection as a reference, an upper-bound, lower-bound, and intermediate value of the theoretical numbers of target MEls could be estimated. The lower-bound for target MEls was defined as a MEI sequence that contains the sequence the gRNA binds to with $100 \%$ (or 23bp) matched sequence, the intermediate bound allows for $\leq 3 \mathrm{bp}$ mismatch or gap between the gRNA sequence and the matched MEl sequence, and the upper bound is a gRNA that aligns with more than $60 \%$ matched sequence (or $\geq 14 \mathrm{bp}$ ) to the MEI.

In vitro transcription of guide RNA and Cas9 ribonucleoprotein formation

Single stranded DNA oligos were designed using the EnGen sgRNA Designer tool (https://sgrna.neb.com/\#!/sgrna, New England Biolabs) and purchased from IDT (Integrated DNA Technologies) to be used in the EnGen sgRNA Synthesis Kit (New England Biolabs, E3322S). Lyophilized oligos were resuspended in molecular biology grade water to a concentration of $100 \mathrm{uM}$, and 1:10 dilutions were made for working stocks. Each reaction was set up containing 10uL of EnGen 2X sgRNA Reaction Mix (S. pyrogenes), 0.5uL of 100mM DTT, 2.5uL of 10uM oligonucleotide, 2uL of EnGen sgRNA Enzyme Mix, and brought to 20uL total with PCR grade water. The reactions were incubated at $37^{\circ} \mathrm{C}$ for 30 minutes to 1 hour. To degrade leftover DNA oligonucleotides, the reaction volume was adjusted to 50uL using PCR grade water, and 2uL of DNase (New England Biolabs, E3322S) was added to the sample and incubated for 15 minutes at $37^{\circ} \mathrm{C}$. The sgRNA was purified by adding 200uL of Trizol and 50uL of chloroform to the sample, vortexed to mix and centrifuged at 20,000xg at room temperature. The aqueous layer was removed and placed into a new $1.5 \mathrm{~mL}$ microcentrifuge tube and extracted again using 50uL of chloroform. The aqueous layer was removed and placed into a new $1.5 \mathrm{~mL}$ microcentrifuge tube and ethanol precipitated in 2 volumes of $100 \%$ ethanol, and sodium acetate was added to a final concentration of $0.3 \mathrm{M}$. The sample was centrifuged at max speed at $4^{\circ} \mathrm{C}$ for 30 minutes. The RNA pellet was washed with $70 \%$ ethanol, air dried, and resuspended in 10uL of PCR grade water. RNA concentration was measured using the Qubit RNA BR Assay Kit (ThermoFisher, Q10211). Fresh guide RNA was transcribed for every experiment, and prepared no more than a day in advance.

The Cas9 ribonucleoprotein (RNP) was formed by combining 850ng of in vitro transcribed guide RNA, 1uL of a 1:5 dilution of Alt-R S.p.Cas9 Nuclease V3 (Integrated DNA Technologies, 1081058), and $1 \mathrm{X}$ Cutsmart buffer (New England Biolabs, B7204S) in a total of 30uL. To allow for sufficient RNP formation, the reaction was incubated at room temperature for 20 minutes.

\section{Cas9 enrichment for L1Hs on a MinION flow cell}

To perform a Cas9 sequencing enrichment for L1Hs, a modified Cas9 enrichment experiment was performed $^{44}$. Three identical aliquots of 10 ug of GM12878 genomic DNA were exhaustively dephosphorylated in a total volume of 40uL, with 1X Cutsmart buffer, 6uL of Quick CIP (New England Biolabs, M0525S), 10ug of gDNA, and $\mathrm{H} 2 \mathrm{O}$ for 30 minutes at $37^{\circ} \mathrm{C}$. The Quick CIP was heat inactivated at $80^{\circ} \mathrm{C}$ for 20 minutes. 20uL of RNP (Cas9 + gRNA) was added to the 
reaction along with $2 \mathrm{uL}$ of Taq polymerase (New England Biolabs, M0273L) and 1.5uL of 10mM dATP. The reaction was mixed by tapping and incubated at $37^{\circ} \mathrm{C}$ for 30 minutes for Cas 9 cleavage, and $72^{\circ} \mathrm{C}$ for 10 minutes for monoadenylation. Following monoadenylation, each reaction was combined with 50uL of ligation mix: 25uL Ligation Buffer (LNB; Oxford Nanopore Technologies, SQK-LSK109), 5uL of Adapter Mix X (AMX; Oxford Nanopore Technologies, SQK-LSK109), 12.5uL of T4 DNA ligase (New England Biolabs, M0202M), and 5uL of nuclease-free water. The nanopore adapters were ligated to the genomic DNA at room temperature for 30 minutes on a tube rotator. Once completed, the ligations were diluted with 1 volume of 1 X TE buffer (100uL). 60uL of SPRI beads (Beckman Coulter, B23317) were added to the adapterr ligated samples and incubated at room temperature for 10 minutes with rotation and for another 5 minutes without rotation. Beads were immobilized using a magnet and the supernatant was removed. Immobilized beads were resuspended with 200ul of room temperature L fragment buffer (LFB; Oxford Nanopore Technologies, SQK-LSK109. At this step, the resuspendend beads from the three samples were pooled into one Eppendorf tube. The magnet was applied again to immobilize the beads and remove the supernatant and the wash was repeated. Washed samples were pulse spun on a tabletop centrifuge for 1 second to collect beads at the bottom. Residual LFB was aspirated with a pipette. Beads were resuspended in 16.8uL of Elution Buffer (EB; Oxford Nanopore Technologies, SQK-LSK109) and incubated at room temperature for 10 minutes. Following the elution, the magnet was applied and the supernatant was collected and placed into a sterile Eppendorf tube. In some sample preparations, the adapter ligated library eluted in the last step may be viscous and the beads will resist immobilization on the magnet. A maximum speed centrifugation step prior to applying the magnet will help to immobilize the beads. Once the supernatant was separated from the beads into a sterile Eppendorf tube, 26uL of Sequencing Buffer (SQB; Oxford Nanopore Technologies, SQK-LSK109) was added and placed on ice until the sequencing flow cell was prepared. Immediately prior to loading of the sample, 0.5uL of Sequencing Tether (SQT, Oxford Nanopore Technologies, SQK-LSK109) was added along with 9.5 uL of Loading Beads (LB; Oxford Nanopore Technologies, SQK-LSK109). The sample was mixed evenly by pipetting with a p20 and loaded onto the sequencing platform.

Pooled Cas9 enrichment for L1Hs, AluYb, AluYa, SVA_F, and SVA_E in GM12878 (MinION)

Five parallel Cas9 enrichment experiments were performed for the five MEl subfamilies in GM12878 for a pooled sequencing run. Five separate aliquots of 10 ug of genomic DNA were dephosphorylated in 40uL total (30uL of gDNA, 4uL of 10X CutSmart, 6uL of Quick CIP) for 25 minutes at $37^{\circ} \mathrm{C}$ then heat inactivated at $80^{\circ} \mathrm{C}$ for 5 minutes. Following the heat inactivation, each dephosphorlyated genomic DNA sample was combined with 20uL of Cas9 RNP, 1uL of Taq polymerase, and $1 \mathrm{uL}$ of $10 \mathrm{mM}$ dATP. After briefly mixing by tapping, the reaction was incubated at $37^{\circ} \mathrm{C}$ for 30 minutes to enable Cas 9 cleavage, then incubated to $75^{\circ} \mathrm{C}$ for monoadenylation by Taq polymerase. The Cas 9 digested and monoadenylated samples were pooled into the ligation reaction (164uL of Custom LNB, 10uL of AMX, 20uL of T4 DNA ligase, and $164 \mathrm{uL}$ of nuclease-free water) and rotated at room temperature for 30 minutes. One volume of $1 \mathrm{X}$ TE buffer was added to the ligation and mixed by inversion approximately 10 times, or 
until evenly mixed. 0.3X sample volume of SPRI beads (394.8uL) was added and incubated at room temperature with rotation for 5 minutes. The beads were immobilized using a magnet and washed twice with 100uL of room temperature LFB. After the final wash, the beads were pulse spun for 1 second in a table top centrifuge, immobilized on a magnet, and residual LFB was removed. The washed beads were eluted in $13 \mathrm{uL}$ of EB for 10 minutes at room temperature and removed using a magnet. The supernatant was collected and combined with $26 \mathrm{uL}$ of SQB. The library was incubated on ice until the flow cell was prepared. 0.5uL of SQT and 9.5uL of LB were added to the library before the loading onto the flow cell.

\section{Cas9 enrichment for single MEI subfamily on a Flongle flow cell}

10ug of purified genomic DNA was dephosphorylated using 4uL of Quick CIP in 1X Cutsmart buffer and brought to a total reaction volume of $40 \mathrm{uL}$, then incubated for 30 minutes at $37^{\circ} \mathrm{C}$. The sample was then incubated at $80^{\circ} \mathrm{C}$ for 5 minutes to inactivate the Quick CIP. 1uL of Taq polymerase, $1 \mathrm{uL}$ of $10 \mathrm{mM} \mathrm{dATP}$, and $20 \mathrm{uL}$ of the corresponding Cas9 RNP (targeting L1Hs, AluYb, AluYb, SVA_F, or SVA_E), was added to the dephosphorlyated genomic DNA, gently mixed, and incubated at $37^{\circ} \mathrm{C}$ for 30 minutes, followed by a 10 minute incubation at $75^{\circ} \mathrm{C}$. The sample was added to the ligation solution (25uL of custom LNB, 6uL of T4 DNA ligase, 5uL of AMX, and nuclease-free water to $100 \mathrm{uL}$ total), and incubated at room temperature for 20 minutes with rotation. The ligation was mixed with 1 volume (100uL) of 1 X TE buffer and mixed by inversion approximately 10 times, or until evenly mixed. SPRI beads were added to a final $0.3 \mathrm{X}(60 \mathrm{uL})$ to the sample volume $(200 \mathrm{uL})$ and the sample was rotated at room temperature for 5 minutes. The SPRI beads were immobilized on a magnet and washed twice with 100uL of room temperature LFB. After the final wash, the beads were pulse spun for 1 second on tabletop centrifuge and residual LFB was removed. The beads were resuspended in 9uL of EB and incubated at room temperature for 10 minutes. After the elution, the beads were immobilized on a magnet and the supernatant was transferred to a new $1.5 \mathrm{~mL}$ microcentrifuge tube. 13uL of SQB was added to the supernatant and this library was placed on ice until the flow cell was prepared. Before loading the sample onto the flow cell, 0.5uL of SQT and 9.5uL of LB were added and mixed by gentle tapping.

\section{Cas9 enrichment for L1Hs in trio (MinION)}

To detect L1Hs in the lymphoblastoid trio cells (GM12878/91/92), a modified Cas9 enrichment assay, originally described by Gilpatrick et al. 2020, was performed. 10ug of genomic DNA for each genome (30ug total) was exhaustively dephosphorylated using 6uL Quick CIP for 45 minutes at $37^{\circ} \mathrm{C}$, and heat inactivated at $80^{\circ} \mathrm{C}$ for 20 minutes. 20uL of the RNP (Cas9 and sgRNA) was added to the dephosphorylated genomic DNA along with $2 \mathrm{uL}$ of Taq DNA polymerase and $1.5 \mathrm{uL}$ of $10 \mathrm{mM}$ dATP. The reaction was incubated at $37^{\circ} \mathrm{C}$ for 30 minutes, then $72^{\circ} \mathrm{C}$ for 10 minutes. Each genomic DNA reaction was combined with an equal volume (50uL) of ligation mix for nanopore adapter ligation: 25uL Ligation Buffer (Oxford Nanopore Technologies, EXP-NBD104), 5uL of AMII (Adapter Mix II; Oxford Nanopore Technologies, EXP-NBD104), 12.5uL of T4 DNA ligase, 2.5uL of the barcode (NB01/02/03; Oxford Nanopore 
Technologies, EXP-NBD104), and 2.5uL of nuclease-free water, and incubated at room temperature for 30 minutes on a tube rotator. After adapter ligation, an equal volume of $1 \mathrm{X}$ TE buffer was added to the reaction, and SPRI beads were added to a final $0.3 \mathrm{X}(\sim 60 \mathrm{uL})$. The library was incubated at RT for 10 minutes with rotation, and 10 minutes without rotation for a total of 20 minutes to allow for DNA binding to the SPRI beads. The beads were washed twice with 200uL of L Fragment Buffer (LFB; Oxford Nanopore Technologies, EXP-NBD104). The uniquely barcoded samples were pooled by combining the resuspended beads in the first wash, then washed again. The washed beads were resuspended in 16.8uL of the Elution Buffer (EB; Oxford Nanopore Technologies, EXP-NBD104). Resuspended beads were incubated at room temperature for 10 minutes. Following the incubation, the beads were collected with a magnet and the supernatant collected into a separate $1.5 \mathrm{~mL}$ microcentrifuge tube and placed on ice. The sample was prepared for sequencing by adding 26uL of Sequencing Buffer (SQB; Oxford Nanopore Technologies, EXP-NBD104) and kept on ice while the flow cell was primed. 0.5uL of Sequencing Tether (SQT; Oxford Nanopore Technologies, EXP-NBD104) and 9.5uL of Loading Beads (LB; Oxford Nanopore Technologies, EXP-NBD104) were added after flow cell priming, before the sample was loaded onto the flow cell.

Nanopore flow cell preparation, sequencing, base-calling, and cleavage-site analysis

A MinION flow cell was purchased from Oxford Nanopore Technologies and stored at $4^{\circ} \mathrm{C}$ per manufacturer's instructions. The Ligation Sequencing Kit (SQK-LSK109) and Native Barcoding Kit (EXP-NBD104) were used to prepare the pooled libraries. Upon arrival and prior to usage, MinION flow cell QC was performed using the MinKNOW software. No appreciable loss of active pores was noted during storage. Prior to loading the library, the MinION was flushed with $800 u L$ of FLB in the priming port, followed by priming of the flow cell with 200uL of 0.5x SQB diluted with water..

Flongle flow cells were purchased from Oxford Nanopore Technologies in batches and stored at $4^{\circ} \mathrm{C}$. Flow cells were $Q C^{\prime} d$ upon arrival and the number of active pores was noted. Flongles to be used in sequencing were QC'd immediately before use to assess pore loss during the storage period.

Base-calling was processed by Guppy 4.0.15 (Oxford Nanopore Technologies) using the high accuracy, modified base model (dna_r9.4.1_450bps_modbases_dam-dcm-cpg_hac.cfg). Porechop ${ }^{76}$ was used to trim nanopore adapters and barcodes from the reads with Q-score $>7$, as well as demultiplex the reads in the pooled sample MinION run.

To determine cut site preferences, reads were aligned to the consensus sequence of each mobile element class that were investigated; L1Hs, AluYa5, AluYb8, SVA_E and SVA_F. Only the first 80 base pairs of each read were used for alignment to focus on the cut site region and to ensure that the beginning of the reads aligned correctly. Pairwise alignments were performed using the Biopython Bio.Align package ${ }^{77}$ with FASTA files as input. Each read was aligned to the mobile element consensus sequence as well as the reverse complement to determine sequence orientation. To obtain high-confidence alignments, strict gap penalties were enforced (open gap:-10, extend gap:-5). In order for an alignment to be considered for cut-site analysis, it 
had to meet two criteria; the alignment had to start at the very first base of the read, and needed to have an alignment score of at least 100. The 5' ends of reads meeting this criteria were then used to estimate cleavage site location.

\section{Nano-Pal for detection and refinement of MEls from nanopore Cas9 enrichment}

To resolve both the reference and non-reference MEI signals from nanopore Cas 9 enrichment, we developed a computational pipeline, Nano-Pal, to analyze the nanopore reads and customized it for different MEI subfamilies (Fig.1c,d). Information of the potential targeting MEI signals was obtained by Nano-Pal scanning through both sides (100bp bin size) of all quality-passed nanopore raw reads using $B L A S T n^{78,79}$. Next, it aligned the reads to the reference genome (GRCh38) using minimap ${ }^{80}$ and discarded reads with low mapping quality $(M A P Q<10)$. The aligned reads were screened by RepeatMasker ${ }^{64}$ and the pre-masking module in PALMER ${ }^{29}$ to bin them into different categories: reads with reference MEl signals, reads with non-reference MEI signals, and off-target reads. All reads that were reported by the PALMER pre-masking module fell into the on-target non-reference MEl category. If reads were not reported by PALMER, but were annotated by RepeatMasker, they fell into the reference MEI category. They will be further classified as on-target, close-target, and off-target reads depending on where they mapped to the reference regions. For L1Hs experiments, the reads mapped to the reference L1PA are considered as close-target and the ones mapped to other reference $\mathrm{L} 1$ are considered as off-target. For AluY experiments, the close-target reads are those that mapped to other reference AluY besides AluYb and AluYa and the off-target reads are defined when mapped to other reference Alu elements besides the reference AluY. For SVA experiments, the close-target reads are the ones mapped to other reference SVAs besides SVA_F and SVA_E and no off-target reads were defined when they have the MEI signals. Any remaining reads with no MEI signals were classified as off-target reads as well. The reads in the first and second category were then clustered into non-reference MEls and reference MEls, respectively. Nano-Pal was performed for each Flongle and MinION flow cell separately for GM12878, GM12891, and GM12892.

\section{GM12878 trio data, reference genome, and reference MEl information}

We obtained Pacific Bioscience (PacBio) long-read CLR sequencing data from Audano et al. $2019^{57}$ for the GM12878 genome (50x coverage). The 30x Illumina NovaSeq sequencing data for GM12878 and the related samples (GM12891 and GM12892) were obtained from the 1000 Genomes project phase 3 sample set, which were generated at the New York Genome Center (

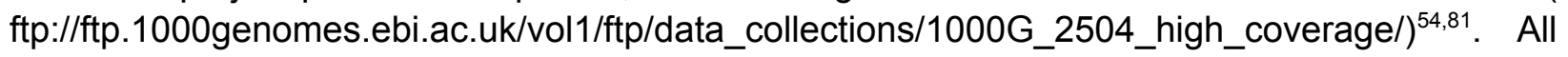
analyses in this project were carried out using the GRCh38 (GRCh38+decoy) reference genome obtained from the 1000 Genomes Project (ftp://ftp.1000genomes.ebi.ac.uk/vol1/ftp/technical/reference/GRCh38_reference_genome/). Information of reference MEls, including the five target subfamilies, were obtained from RepeatMasker ${ }^{64}$. 
Enhanced PALMER for resolving non-reference MEls from whole-genome long-read sequencing

We developed an enhanced version of PALMER (Pre-mAsking Long reads for Mobile Element inseRtion $)^{29}$ in this study to detect non-reference MEls across the long-read sequenced genomes (https://github.com/mills-lab/PALMER). Reference-aligned BAM files from long-read technology were used as input. Known reference repetitive sequences (L1s, Alus or SVAs) were used to pre-mask portions of individual reads that aligned to these repeats and also utilized in the Nano-Pal pipeline. After the pre-masking process, PALMER searched subreads against a library of consensus mobile element sequences within the remaining unmasked sequences and identified reads with a putative insertion signal (including $5^{\prime}$ inverted L1 sequences, if available) as supporting read candidates. PALMER opens bins 5' upstream and 3' downstream of the putative insertion sequence for each read and identifies hallmarks of mobile elements, such as target site duplication (TSD) motifs, transductions, and poly(A) tract sequences. All supporting reads are clustered at each locus and those with a minimum number of supporting events are reported as putative insertions.

To improve the accuracy of non-reference MEl sequences derived from individual subreads, which tend to have lower per-read base-pair accuracy, local sequence alignments and error correction strategies were performed. Error correction was conducted by CANU ${ }^{82}$ (ver2.2) using default parameters on the subreads with MEI signals reported by PALMER, allowing the generation of error-corrected reads that served as inputs for local realignment using minimap2. A second-pass of the PALMER pipeline then was executed using these locally aligned error-corrected reads to generate a high-confidence call set of germline non-reference MEls. $\mathrm{CAP}^{83}$ was used with default parameters to assemble all MEI sequences reported by the second-pass of the PALMER pipeline to generate a high-confidence consensus contig for each non-reference MEl event.

\section{MEI callsets in orthogonal short-read and long-read data}

As there is no public long-read data available for GM12891 and GM12892, we used the Mobile Element Locator Tool (MELT) to identify non-reference MEls in the short-read Illumina sequencing data for the GM12878 trio ${ }^{27,65}$ as a benchmark set in the trio analysis. We applied the enhanced version of PALMER and carried out the non-reference MEI calling in GM12878. To generate a more comprehensive callset of non-reference MEls in GM12878, the Phased Assembly Variant (PAV) caller was included (https://github.com/EichlerLab/pav), which can discover genetic variants based on a direct comparison between two sequence-assembled haplotypes and the human reference genome ${ }^{40}$. The callset by PAV for GM12878 was generated from the PacBio HIFI sequencing data after haplotype-assembly. A 'PacBio-MEl' callset in GM12878 was generated by applying the union set of the mapping-based PALMER callset and the assembly-based PAV callset, both of which resolved the MEls from PacBio long-read sequencing data. The details of MEI merging and subfamily defining strategy for the two approaches are described in a prior study ${ }^{40}$. 
Inspection and validation of nanopore-specific non-reference MEls

All non-reference MEI calls were further intersected with the PacBio-MEl set and classified as known non-reference calls and potential nanopore-specific non-reference calls. A filtering module, an empirical curation of read-depth (<2-fold difference) within the $500 \mathrm{bp}$ bin of the insertion site from public data ${ }^{57}$, and manual inspection were applied to exclude false-positive (FP) signals in the nanopore-specific non-reference MEls. All potential nanopore-specific non-reference MEls were classified into three categories: a) true positive (TP) non-reference event, b) FP non-reference event, and c) an ambiguous event (Supplementary Table 7). The category was further defined as TP missed by the PacBio sequencing data, TP missed by the PacBio mapped-based and assembly-based pipelines but with PacBio read signals, or TP redundant with the called non-reference one. Category (b) was broken down into three subcategories; FP redundant with the called reference event, FP targeting on the other off-target reference repeat, or ambiguous. All subcategories in (a), and the first (b) subcategory, were on-target reads with or without correct annotations. For example, one FP nanopore-specific non-reference call originated from reads targeted to reference MEls, yet was categorized as non-reference due to a mapping error introduced by flanking structural variations (deletions, duplications, or inversions) (Supplementary Table 7). A recurrence plot analysis was further employed for further in-depth validation. For the recurrence plot analysis, a region of one sequence (X-axis) is compared to another sequence (Y-axis) and small (i.e. 10 bp) segments that are identical between the two sequences are denoted with a plotted point. Thus, a continuous diagonal line comprising multiple points indicates portions of the compared sequences that are identical. By comparison, gaps and shifts from the diagonal denote an insertion or deletion in one sequence relative to the other.

\section{Non-reference MEls captured by nanopore Cas9 enrichment sequencing in GM12878}

The flow cell runs for each MEl subfamily were merged to investigate the read coverage enrichment performance and generate the final non-reference MEls in GM12878. For the saturation analysis, the flow cells were ranked by the number of on-target reads. Reads from the flow cells were then added and merged, one flow cell at a time, based on the above ranking. Non-reference calls for L1Hs, AluYb, AluYa, SVA_F, and SVA_E were resolved by Nano-Pal and our validation process after every merging instance. By merging all batches for each subfamily, a final non-reference MEl callset in GM12878 captured by nanopore Cas9 enrichment approach was produced.

\section{Data availability}

The nanopore sequencing data for the Cas9 targeted enrichment of MEls in this study are available in the SRA repository under BioProject accession PRJNA699027. 


\section{Code availability}

All script and pipelines in this publication, including Nano-Pal, are available on GitHub: https://github.com/Boyle-Lab/NanoPal-and-Cas9-targeted-enrichement-pipelines.

The enhanced version of PALMER is available at https://github.com/mills-lab/PALMER.

\section{Acknowledgements}

We thank Dr. Scott Devine, Dr. Qihui Zhu, and Dr. Charles Lee for providing the MELT callset for GM12892 and GM12891. We thank Jixin Guan for their help with improving the performance of the PALMER software. This research was supported by the National Institutes for Health (NIH) under award no. R21HG011493 to A.P.B. and R.E.M.. T.M. was supported by T32GM007544. C.C. was supported by the University of Michigan Rackham Merit Fellowship and the Training Program in Bioinformatics (T32GM070449).

\section{Author Contributions}

A.P.B., R.E.M., W.Z., and T.M. conceived the project. J.S. established and cultured cell lines and isolated gDNA. T.M. performed the Cas9 targeted enrichment and nanopore sequencing. W.Z. developed the NanoPal pipeline and PALMER software. W.Z., C.C., and C.M. performed computational analysis. All authors guided the data analysis strategy. A.P.B., R.E.M., W.Z., and T.M. wrote the manuscript. All authors edited the manuscript. All authors read and approved the final manuscript.

\section{Competing Interests statement}

The authors declare no competing interests.

\section{Reference}

1. Smit, A. F. Interspersed repeats and other mementos of transposable elements in mammalian genomes. Curr. Opin. Genet. Dev. 9, 657-663 (1999).

2. Lander, E. S. et al. Initial sequencing and analysis of the human genome. Nature 409, 860-921 (2001).

3. Deininger, P. Alu elements: know the SINEs. Genome Biol. 12, 236 (2011).

4. Ostertag, E. M., Goodier, J. L., Zhang, Y. \& Kazazian, H. H., Jr. SVA elements are nonautonomous retrotransposons that cause disease in humans. Am. J. Hum. Genet. 73, 1444-1451 (2003). 
5. Kazazian, H. H., Jr \& Moran, J. V. Mobile DNA in Health and Disease. N. Engl. J. Med. 377, 361-370 (2017).

6. Brouha, B. et al. Hot L1s account for the bulk of retrotransposition in the human population. Proc. Natl. Acad. Sci. U. S. A. 100, 5280-5285 (2003).

7. Sassaman, D. M. et al. Many human L1 elements are capable of retrotransposition. Nat. Genet. 16, 37-43 (1997).

8. Beck, C. R. et al. LINE-1 retrotransposition activity in human genomes. Cell 141, 1159-1170 (2010).

9. Scott, E. C. et al. A hot L1 retrotransposon evades somatic repression and initiates human colorectal cancer. Genome Res. 26, 745-755 (2016).

10. Kazazian, H. H., Jr et al. Haemophilia A resulting from de novo insertion of L1 sequences represents a novel mechanism for mutation in man. Nature 332, 164-166 (1988).

11. Lubelsky, Y. \& Ulitsky, I. Sequences enriched in Alu repeats drive nuclear localization of long RNAs in human cells. Nature 555, 107-111 (2018).

12. Aneichyk, T. et al. Dissecting the Causal Mechanism of X-Linked Dystonia-Parkinsonism by Integrating Genome and Transcriptome Assembly. Cell 172, 897-909.e21 (2018).

13. Jourdy, Y. et al. Reccurrent F8 Intronic Deletion Found in Mild Hemophilia A Causes Alu Exonization. Am. J. Hum. Genet. 102, 199-206 (2018).

14. Evrony, G. D., Lee, E., Park, P. J. \& Walsh, C. A. Resolving rates of mutation in the brain using single-neuron genomics. Elife 5, (2016).

15. Upton, K. R. et al. Ubiquitous L1 mosaicism in hippocampal neurons. Cell 161, 228-239 (2015).

16. Coufal, N. G. et al. L1 retrotransposition in human neural progenitor cells. Nature 460, $1127-1131$ (2009).

17. Muotri, A. R. et al. Somatic mosaicism in neuronal precursor cells mediated by L1 retrotransposition. Nature 435, 903-910 (2005). 
18. Zhu, X. et al. Machine learning reveals bilateral distribution of somatic L1 insertions in human neurons and glia. doi:10.1101/660779.

19. McConnell, M. J., Moran, J. V., Abyzov, A. \& Akbarian, S. Intersection of diverse neuronal genomes and neuropsychiatric disease: The Brain Somatic Mosaicism Network. (2017).

20. Diehl, A. G., Ouyang, N. \& Boyle, A. P. Transposable elements contribute to cell and species-specific chromatin looping and gene regulation in mammalian genomes. Nat. Commun. 11, 1796 (2020).

21. Zhang, Y. et al. Transcriptionally active HERV-H retrotransposons demarcate topologically associating domains in human pluripotent stem cells. Nat. Genet. 51, 1380-1388 (2019).

22. Choudhary, M. N. K. et al. Co-opted transposons help perpetuate conserved higher-order chromosomal structures. doi:10.1101/485342.

23. Iskow, R. C. et al. Natural mutagenesis of human genomes by endogenous retrotransposons. Cell 141, 1253-1261 (2010).

24. Steranka, J. P. et al. Transposon insertion profiling by sequencing (TIPseq) for mapping LINE-1 insertions in the human genome. Mob. DNA 10, 8 (2019).

25. Huang, C. R. L. et al. Mobile interspersed repeats are major structural variants in the human genome. Cell 141, 1171-1182 (2010).

26. Erwin, J. A. et al. L1-associated genomic regions are deleted in somatic cells of the healthy human brain. Nature Neuroscience vol. 19 1583-1591 (2016).

27. Gardner, E. J. et al. The Mobile Element Locator Tool (MELT): population-scale mobile element discovery and biology. Genome Res. 27, 1916-1929 (2017).

28. Kvikstad, E. M., Piazza, P., Taylor, J. C. \& Lunter, G. A high throughput screen for active human transposable elements. BMC Genomics 19, 115 (2018).

29. Zhou, W. et al. Identification and characterization of occult human-specific LINE-1 insertions using long-read sequencing technology. Nucleic Acids Res. 48, 1146-1163 (2020). 
30. Thung, D. T. et al. Mobster: accurate detection of mobile element insertions in next generation sequencing data. Genome Biol. 15, 488 (2014).

31. Wu, J. et al. Tangram: a comprehensive toolbox for mobile element insertion detection. BMC Genomics 15, 795 (2014).

32. Lee, E. et al. Landscape of somatic retrotransposition in human cancers. Science 337 , 967-971 (2012).

33. Chaisson, M. J. P. et al. Multi-platform discovery of haplotype-resolved structural variation in human genomes. Nat Commun 10: 1784. (2019).

34. Kidd, J. M. et al. A human genome structural variation sequencing resource reveals insights into mutational mechanisms. Cell 143, 837-847 (2010).

35. Beck, C. R., Garcia-Perez, J. L., Badge, R. M. \& Moran, J. V. LINE-1 elements in structural variation and disease. Annu. Rev. Genomics Hum. Genet. 12, 187-215 (2011).

36. Ha, H., Loh, J. W. \& Xing, J. Identification of polymorphic SVA retrotransposons using a mobile element scanning method for SVA (ME-Scan-SVA). Mob. DNA 7, 15 (2016).

37. Flasch, D. A. et al. Genome-wide de novo L1 Retrotransposition Connects Endonuclease Activity with Replication. Cell 177, 837-851.e28 (2019).

38. Chin, C.-S. et al. Nonhybrid, finished microbial genome assemblies from long-read SMRT sequencing data. Nat. Methods 10, 563-569 (2013).

39. Jain, M. et al. Nanopore sequencing and assembly of a human genome with ultra-long reads. Nat. Biotechnol. 36, 338-345 (2018).

40. Ebert, P. et al. De novo assembly of 64 haplotype-resolved human genomes of diverse ancestry and integrated analysis of structural variation. bioRxiv (2020).

41. Zook, J. M. et al. A robust benchmark for detection of germline large deletions and insertions. Nat. Biotechnol. 38, 1347-1355 (2020).

42. Chu, C., Zhao, B., Park, P. J. \& Lee, E. A. Identification and Genotyping of Transposable Element Insertions From Genome Sequencing Data. Curr. Protoc. Hum. Genet. 107, e102 
(2020).

43. Ewing, A. D. et al. Nanopore sequencing enables comprehensive transposable element epigenomic profiling. doi:10.1101/2020.05.24.113068.

44. Gilpatrick, T. et al. Targeted nanopore sequencing with Cas9-guided adapter ligation. Nat. Biotechnol. 38, 433-438 (2020).

45. Wang, H. et al. SVA elements: a hominid-specific retroposon family. J. Mol. Biol. 354, 994-1007 (2005).

46. Bennett, E. A. et al. Active Alu retrotransposons in the human genome. Genome Res. 18, 1875-1883 (2008).

47. Boissinot, S., Chevret, P. \& Furano, A. V. L1 (LINE-1) retrotransposon evolution and amplification in recent human history. Mol. Biol. Evol. 17, 915-928 (2000).

48. Karamitros, T. \& Magiorkinis, G. Multiplexed Targeted Sequencing for Oxford Nanopore MinION: A Detailed Library Preparation Procedure. Methods Mol. Biol. 1712, 43-51 (2018).

49. Gabrieli, T. et al. Selective nanopore sequencing of human BRCA1 by Cas9-assisted targeting of chromosome segments (CATCH). Nucleic Acids Res. 46, e87 (2018).

50. Giesselmann, P. et al. Analysis of short tandem repeat expansions and their methylation state with nanopore sequencing. Nat. Biotechnol. 37, 1478-1481 (2019).

51. Dausset, J. et al. Centre d'etude du polymorphisme humain (CEPH): collaborative genetic mapping of the human genome. Genomics 6, 575-577 (1990).

52. International HapMap Consortium. The International HapMap Project. Nature 426, 789-796 (2003).

53. Mills, R. E. et al. Mapping copy number variation by population-scale genome sequencing. Nature 470, 59-65 (2011).

54. Consortium, T. 1000 G. P. \& The 1000 Genomes Project Consortium. A global reference for human genetic variation. Nature vol. 526 68-74 (2015).

55. Zook, J. M. et al. Extensive sequencing of seven human genomes to characterize 
benchmark reference materials. Scientific data vol. 3160025 (2016).

56. Zook, J. M. et al. Integrating human sequence data sets provides a resource of benchmark SNP and indel genotype calls. Nat. Biotechnol. 32, 246-251 (2014).

57. Audano, P. A. et al. Characterizing the Major Structural Variant Alleles of the Human Genome. Cell 176, 663-675.e19 (2019).

58. Mills, R. E., Bennett, E. A., Iskow, R. C. \& Devine, S. E. Which transposable elements are active in the human genome? Trends Genet. 23, 183-191 (2007).

59. Moran, J. V. et al. High frequency retrotransposition in cultured mammalian cells. Cell 87, 917-927 (1996).

60. Ran, F. A. et al. Genome engineering using the CRISPR-Cas9 system. Nat. Protoc. 8, 2281-2308 (2013).

61. Sternberg, S. H., Redding, S., Jinek, M., Greene, E. C. \& Doudna, J. A. DNA interrogation by the CRISPR RNA-guided endonuclease Cas9. Nature 507, 62-67 (2014).

62. Cong, L. et al. Multiplex genome engineering using CRISPR/Cas systems. Science 339, 819-823 (2013).

63. Hsu, P. D. et al. DNA targeting specificity of RNA-guided Cas9 nucleases. Nat. Biotechnol. 31, 827-832 (2013).

64. Smit, A. F. A., Hubley, R. \& Green, P. 2015 RepeatMasker Open-4.0. (2013).

65. Sudmant, P. H. et al. An integrated map of structural variation in 2,504 human genomes. Nature 526, 75-81 (2015).

66. Zhao, X., Weber, A. M. \& Mills, R. E. A recurrence-based approach for validating structural variation using long-read sequencing technology. Gigascience 6, 1-9 (2017).

67. Hehir-Kwa, J. Y. et al. A high-quality human reference panel reveals the complexity and distribution of genomic structural variants. Nat. Commun. 7, 12989 (2016).

68. Stangl, C. et al. Partner independent fusion gene detection by multiplexed CRISPR-Cas9 enrichment and long read nanopore sequencing. Nat. Commun. 11, 2861 (2020). 
69. Niu, Y. et al. Genome-wide analysis of mobile element insertions in human genomes. bioRxiv (2021).

70. Miga, K. H. et al. Telomere-to-telomere assembly of a complete human $\mathrm{X}$ chromosome. Nature 585, 79-84 (2020).

71. Bennetzen, J. L. Transposable element contributions to plant gene and genome evolution. Plant Mol. Biol. 42, 251-269 (2000).

72. Yu, T. et al. A benchmark and an algorithm for detecting germline transposon insertions and measuring de novo transposon insertion frequencies. Nucleic Acids Res. (2021) doi:10.1093/nar/gkab010.

73. Miller, S. A., Dykes, D. D. \& Polesky, H. F. A simple salting out procedure for extracting DNA from human nucleated cells. Nucleic Acids Res. 16, 1215 (1988).

74. Jurka, J. Repeats in genomic DNA: mining and meaning. Curr. Opin. Struct. Biol. 8, 333-337 (1998).

75. Marçais, G. \& Kingsford, C. A fast, lock-free approach for efficient parallel counting of occurrences of k-mers. Bioinformatics 27, 764-770 (2011).

76. Wick, R. R., Judd, L. M., Gorrie, C. L. \& Holt, K. E. Completing bacterial genome assemblies with multiplex MinION sequencing. Microb Genom 3, e000132 (2017).

77. Cock, P. J. A. et al. Biopython: freely available Python tools for computational molecular biology and bioinformatics. Bioinformatics 25, 1422-1423 (2009).

78. Zhang, Z., Schwartz, S., Wagner, L. \& Miller, W. A greedy algorithm for aligning DNA sequences. J. Comput. Biol. 7, 203-214 (2000).

79. Altschul, S. F., Gish, W., Miller, W., Myers, E. W. \& Lipman, D. J. Basic local alignment search tool. J. Mol. Biol. 215, 403-410 (1990).

80. Li, H. Minimap2: pairwise alignment for nucleotide sequences. Bioinformatics 34, 3094-3100 (2018).

81. Byrska-Bishop, M. et al. High coverage whole genome sequencing of the expanded 1000 
Genomes Project cohort including 602 trios. doi:10.1101/2021.02.06.430068.

82. Koren, S. et al. Canu: scalable and accurate long-read assembly via adaptive k-mer weighting and repeat separation. Genome Res. 27, 722-736 (2017).

83. Huang, X. \& Madan, A. CAP3: A DNA sequence assembly program. Genome Res. 9, 868-877 (1999). 